\title{
Los Semilleros como espacios de investigación para el investigador novel
}

\section{The "Incubators" as Research Spaces for the Novice Researcher}

\author{
Nahín Numa-Sanjuan* \\ Universidad Simón Bolívar, Cúcuta, Colombia \\ ORCID: https://orcid.org/0000-0003-2134-6230 \\ Rodolfo Alfonso Márquez Delgado \\ Universidad Pedagógica Experimental Libertador, Táchira, Venezuela \\ ORCID: https://orcid.org/0000-0003-4501-074X
}

Recibido 11-11-18 Revisado 12-12-18 Aprobado 13-02-19 En línea 14-02-19

\section{*Correspondencia}

Email: nahinnumas@gmail.com

\section{Citar como:}

Numa-Sanjuan, N., \& Márquez, R. (2019). Los Semilleros como espacios de investigación para el investigador novel Propósitos y Representaciones, 7(1), 230-248. Doi: http://dx.doi.org/10.20511/pyr2019.v7n1.289

(C) Universidad San Ignacio de Loyola, Vicerrectorado de Investigación, 2019. 


\section{Resumen}

Este artículo de carácter empírico e interpretativo tiene como objetivo ofrecer un aporte a la discusión de los Semilleros como espacios investigativos en la formación del investigador novel. Es producto de reflexiones realizadas por los autores sobre la temática y de las discusiones realizadas con jóvenes de pregrado y postgrado que aspiran ser investigadores en el campo de la educación. Se sustenta en un marco referencial relacionado con: a) la investigación como Proceso sistemático que se aprende y enseña y; b) Competencias en Investigación para la formación de los investigadores noveles. Parte del supuesto que investigar en el ámbito de las ciencias de la educación es complejo, sin embargo, ello las hace fértiles y un semillero para obtener competencias investigativas. Se concluye con la propuesta de tres acciones o caminos relacionados con la formación de investigadores nóveles: a) la institucionalización de los semilleros como espacios investigativos; b) la producción de artículos científicos y; c) la articulación de la investigación con el currículo. La expectativa de los autores es que estas reflexiones contribuyan a promover en las instituciones educativas espacios, como los Semilleros de investigación, que permitan que estas superen las brechas investigativas que actualmente existen en Latinoamérica y se consoliden como organizaciones que aprenden y gestionan el conocimiento.

Palabras clave: Formación por competencias; aprendizaje; investigación, enseñanza.

\section{Summary}

This article of empirical and interpretive nature aims to offer a contribution to the discussion of "Incubators" as research spaces in the formation of the novice researcher. It is a product of reflections carried out by the authors on the subject and discussions carried out with undergraduate and graduate young people who aspire to be researchers in the field of education. It is based on a reference framework related to: a) research as a systematic process that is learned and taught and; b) competences in research for the training of novice researchers. It is based on the assumption that to do a research in the field of Education Sciences is complex. However, this makes them fertile and an incubator for research competences. It is concluded with the proposal of three actions or paths associated with the training of novice researchers: to) the institutionalization of "Incubators" as research spaces; (b) the production of scientific papers and; (c) the implementation of the curriculum research. The expectation of the authors is that these reflections contribute to promote educational institutions spaces such as the research "Incubators" allowing them to exceed the research gaps that currently exist in Latin America and that they consolidated as organizations that learn and manage knowledge.

Keywords: Competence-based Training; Learning, Research and Teaching.

\section{Introducción}

La formación en investigación es un proceso que se inicia desde los primeros niveles del sistema educativo. Ello se evidencia en los diferentes planes de estudio: educación inicial, primaria, media hasta la universitaria, donde se indica que los estudiantes desarrollen un pensamiento lógico, critico, reflexivo y sean capaces de analizar y resolver problemas del contexto social en el cual están inmersos.

Lo anterior plantea una situación digna de ser alcanzada en educación y determinante para que en la actualidad se cuente con los más altos niveles de investigadores, no obstante, la realidad es diferente y demuestra ausencia de competencias en investigación, lo cual ha determinado que en muchas instituciones se busquen espacios académicos para fortalecer dichas competencias.

Se está consciente de la necesidad que tienen las instituciones educativas latinoamericanas y más aún las universitarias para que los estudiantes y docentes de pregrado y 
postgrado generen y desarrollen conocimientos. Ello ha sido determinante para organizar los semilleros de investigación como mecanismo para complementar la formación profesional de los estudiantes noveles.

Por lo que antecede, el objetivo de este trabajo discursivo es proponer acciones o caminos institucionales para la formación de investigadores noveles y el desarrollo de sus competencias para la consolidación de estructuras mentales y apropiación del social del conocimiento. Este es de carácter interpretativo, se sustenta en la experiencia de los autores como docentes investigadores y su participación activa en la línea de Investigación sobre Pedagogía que se desarrolla en el Doctorado en Educación de la Universidad Pedagógica Libertador, y en las conversaciones, reflexiones que se han mantenido con estudiantes de pregrado y postgrado sobre esta temática.

El trabajo se organizó en cinco partes: la primera plantea una introducción, la segunda el abordaje problemático, la tercera un breve recorrido referencial, la cuarta acciones o caminos para la formación de investigadores noveles y la quinta ideas conclusivas. Se finaliza con referencias bibliográficas que sustentan el trabajo presentado.

\section{Abordaje Problemático}

La investigación desde siempre ha sido uno de los ámbitos más difíciles de implementar en las universidades. No hay duda que en todas las instituciones han desarrollado asignaturas sobre metodología de la investigación, talleres, programas y líneas de investigación, los cuales tienen como propósito principal motivar a los estudiantes en los procesos investigativos y que adquieran los elementos, teóricos y prácticos, sobre conceptos, métodos y técnicas usualmente empleados en el trabajo de investigación científico. No obstante, estos objetivos se han cumplido parcialmente por cuanto persisten limitaciones para que los estudiantes conjuntamente con sus docentes, realicen investigaciones y aprendan a investigar investigando $\mathrm{y}$ desarrollen competencias como la observación, e indagación, crítica.

Una problemática, que se deriva del planteamiento anterior, es que se carece de cultura en investigación, esta orienta solo a cumplir con el requisito para la elaboración de trabajos de grado a nivel de pregrado y tesis para las Maestrías y Doctorados. En el primero, se supone que los estudiantes deben adquirir ese espíritu investigativo e interés por la investigación, sin embargo, no sucede así, pese a que existen componentes de investigación muy bien estructurados con asignaturas que van desde metodología de la investigación hasta diferentes seminarios que debieran garantizar que los estudiantes desarrollaran una mentalidad crítica, interés por la indagación, cuestionamiento permanente y la posibilidad de crear y recrear el conocimiento. Sin embargo, la experiencia demuestra que en muchas ocasiones esta actividad que debería ser lúdica, de motivación e iniciación en la investigación se convierte en un obstáculo o una tarea difícil de alcanzar (Calderón, 2005).

En el postgrado, la situación es similar, con la única diferencia que, en este nivel, se exige que los estudiantes demuestren el dominio metodológico (Trabajos de grado de maestría) y que se produzcan conocimientos o se innove (Tesis doctorales). No obstante, la realidad es que la mayoría de estos trabajos son descontextualizados y no responden a los problemas nucleares de la realidad y se limitan, por lo general, al cumplimiento de un requisito sin una sólida fundamentación ontoepistemológica que permita trascender lo descriptivo para llegar a la comprensión, explicación e interpretación del objeto de estudio.

Otro nudo problemático lo constituye la escasa formación de competencias en investigación por parte de los docentes lo cual incide en que los estudiantes no logren comprender la importancia del pluralismo epistemológico para abordar las realidades de las ciencias de la educación. Aún persiste en muchos docentes el paradigma cartesiano y no se toma en cuenta la complejidad de la realidad y los factores o eventos que confluyen en la investigación. 


\section{Recorrido Referencial.}

Esta temática es muy amplia, por tanto, se discutirá en forma resumida, los siguientes aspectos: a) la investigación como proceso que se aprende y enseña; b) las competencias en investigación.

\section{La investigación como Proceso sistemático que se aprende y enseña.}

Existen muchas definiciones de investigación presentadas por diferentes autores, entre ellos: Best (1983); AnderEgg (1995,2005); kerlinger (2002); Hernández, Fernández, Baptista (2006). Todos coinciden en que la investigación es un proceso sistemático organizado y sistematizado que pretende resolver un problema, una situación o un evento determinado bajo procesos de rigurosidad científica.

Visto de esta manera, la investigación puede ser aprendida y enseñada mediante la interrelación teoría- práctica lo que facilita que los estudiantes aprendan a investigar investigando y que los docentes no se limiten a enseñar o a transmitir los contenidos por ejemplo de metodología de la investigación o sus componentes, sino los procesos deductivos e inductivos que conllevan a la institución a gestionar sus conocimientos.

Desde esta perspectiva, una institución educativa especialmente la universidad no solo hace ciencia, sino que enseña a hacerla (Salvador Mata, 2009). Lo expresado por el autor mencionado plantea a la institución la necesidad de que sus funciones: docencia, investigación y extensión, se integren como un todo para coadyuvar, mediante procesos de enseñanzaaprendizaje, en la búsqueda y construcción social del conocimiento.

En este contexto, investigar es aprender, es aprehender el conocimiento para aportar con claridad ideas vinculándolas unas con las otras, descubrir hipótesis, armar estrategias y llegar, finalmente, a escribir las ideas propias y los hallazgos de la investigación de manera que otros puedan leerlos, criticarlos para avanzar hacia la socialización del conocimiento (Guido, 2012).

De esta manera, enseñar y aprender la investigación se convierte en el eje transversal del proceso formativo (Gallego \& Rodríguez, 2015), por cuanto ambos términos son procesos indisolubles, el uno depende del otro. Es así que el docente para enseñar investigación no solo transmite conocimientos sino los gestiona de manera que el estudiante adquiere los contenidos: declarativos, procedimentales y actitudinales, interrelacionándolos en todo momento con la práctica de la investigación.

Lo expresado implica que el docente sustente su accionar en el aula como prácticaeducativa-crítica o transformadora, para lo cual, desde el principio, se fundamenta en su experiencia y sujeto productor de saberes, y en el convencimiento que enseñar la investigación no es transferir conocimiento, sino crear las posibilidades para su producción o construcción (Guido, 2012).

Si se asume la investigación como un proceso de aprender a enseñar, entonces es importante mencionar el alcance que tienen los semilleros como espacios o laboratorios que integran la investigación, docencia y extensión y que contribuyen a que el estudiante sea protagonista de su aprendizaje, responsable de construir su propio conocimiento y de adquirir las actitudes y aptitudes propias del ejercicio de la investigación.

\section{Competencias en Investigación para la formación de los investigadores noveles.}

Las competencias tienen diferentes significados y connotaciones. Es un término polisémico relativamente nuevo y complejo que se profundiza en la actualidad debido a que la sociedad del conocimiento ha generado nuevas orientaciones en el proceso formativo de los estudiantes y en 
la reconsideración del papel de las ciencias de la educación, siendo las competencias el eje fundamental para la formación integral del individuo y desarrollo de los diferentes saberes: ser, hacer, conocer y convivir.

En las referencias bibliográficas se encuentran obras de autores que asumen diferentes concepciones sobre las competencias; mencionaremos algunas de ellas:

- Arguelles (1966, pp.23-95) señala que son "actuaciones o capacidades laborales para desempeñar un trabajo";

- Fallows y Steven $(2000$, p. 8) las asocia con "habilidades cognoscitivas para comprender y manipular ideas y pensamientos";

- Le Boterf (200, pp.100-110) se refiere a ellas como" acciones que combinan varios conocimientos y recursos";

- Tobón (2004) las considera como habilidades que integran el saber ser, el saber hacer y el saber conocer para resolver problemas del contexto,

- el Proyecto Tuning (2007) considera que las competencias son la integración de conocimientos como "conocer - comprender", "saber -actuar" y saber cómo ser";

- Barriga y Hernández (2010, p.376) se refiere a ellas como "movilización de saberes cognitivos, emocionales y sociales de la persona para tomar decisiones, formarse juicios, adoptar puntos de vista y asumir los valores";

- El Modelo educativo mexicano (2016, pp. 46-47) las define como la capacidad para "aprender a aprender", "aprender a pensar", a cuestionarse acerca de los diversos fenómenos, sus causas y consecuencias, así como a valorar lo que se aprende en conjunto con otros...”

Se evidencia, por los planteamientos anteriores, que el término de competencia es multidimensional y el uso específico del concepto depende del contexto y de los enfoques sobre educación, pedagogía, investigación, enseñanza, aprendizaje.

En consecuencia, en este trabajo se asume la competencia como la integración o movilización de recursos y capacidades conceptuales, procedimentales y actitudinales que debe poseer un individuo para resolver problemas en diferentes ámbitos de la vida personal, profesional y social lo cual garantiza un desarrollo armónico en la sociedad.

Ahora bien, al referirnos a la formación de competencias investigativas en las ciencias de la educación, el asunto se complejiza no solo por su epistemología sino por el método que se utiliza que es contrapuesto al de las ciencias exactas (Rodríguez, 2005), las cuales han estado orientadas bajo el paradigma positivista que se caracteriza por: monismo metodológico (existe unidad de método y homogeneidad doctrinal), explicación causal (leyes generales hipotéticas), el conocimiento se fundamenta en la lógica, razón y la verificación empírica (Mardones, 1991),

En cambio, en las ciencias de la educación se busca la comprensión e interpretación de los eventos, por lo tanto, no se pretende la búsqueda de leyes generales sino de hechos particulares para poder actuar racionalmente sobre ellos; además, el investigador es objeto y sujeto del proceso investigativo, y, por lo tanto, los fenómenos sociales se comprenden e interpretan desde una visión integral, compleja y dinámica.

A partir de esta perspectiva, en la educación, se genera el conocimiento a partir de las contradicciones encontradas en los problemas que surjan del contexto; se trabaja con el método científico, pero no bajo el modelo lógico - matemático sino el de la razón crítica, por cuanto la sociedad objeto de estudio es también subjetiva y el interés final de la ciencia es emancipador y transformador (Mardones1999). 
La discusión ontoepistemologica que existe en las ciencias de la educación trae como consecuencia dificultades para investigar en ese ámbito, más aún cuando se trata de investigadores noveles que se están formando y que requieren sólidas bases sobre lo que significa investigar en esa ciencia.

Rojas Soriano (1998) señala que para formar un investigador es necesario que éste transite por un proceso mediatizado por fundamentos filosóficos, epistemológicos, metodológicos y técnicos instrumentales que le permita construir conocimientos científicos en educación y los aplique mediante la práctica transformadora.

La investigación debería considerarse como un eje transversal en el currículo o en el plan de estudios de una determinada carrera, por lo que rebasa los límites de una disciplina para desarrollarse en todas ellas de forma inter o multidisciplinaria superando de esta manera la fragmentación o el reduccionismo tradicional del currículo.

Sin duda, la investigación tiene, en las instituciones educativas, un espacio curricular asignado que comienza con metodología de la investigación y continúa con los componentes de seminarios conducentes al grado académico, sin embargo, su tratamiento es memorístico, informativo, descontextualizado y con escasa vinculación con los conocimientos previos obtenidos en el desarrollo de la carrera y con la práctica que debe ser fundamental para aprender a investigar.

La formación de competencias en Investigación es un proceso que se va gestando desde los niveles de primaria y se continúa hasta llegar al pregrado y solidifica en el postgrado. A partir de allí se va formando el investigador, a través de sus aportes investigativos en revistas, su participación en eventos, seminarios, talleres, líneas, semilleros de investigación.

Lo que antecede, es determinante para el desarrollo de las competencias en investigación, más en la actualidad, que, como producto de la sociedad de la información o del conocimiento, se les considera la base fundamental para la formación de un profesional integral sea cual sea su área disciplinaria.

En esta misma línea de pensamiento, Calderón (2005) indica que no se puede esperar que en el pregrado se formen investigadores, sin embargo, es en este nivel, donde se forjan sus actitudes y bases conceptuales, no obstante, en contraposición, se fomenta una posición de rechazo y frustración por dificultades en la praxis pedagógica, y en algunos elementos producto del currículo oculto.

No hay duda de que las competencias de investigación se pueden reafirmar y complementar con los semilleros o líneas de investigación institucionales ya que estos son espacios o comunidades de aprendizaje donde se logra que el estudiante sea autónomo, independiente, creativo, innovador y responsable (Borja, Prada Londoño, et al, 2015).

\section{Acciones o caminos a seguir en el sendero de la formación de los investigadores noveles.}

Esta discusión es producto de la experiencia de los autores como docentes investigadores y de las conversaciones con los estudiantes de pregrado en su proceso de trabajo de grado. A partir de ellas surgieron las siguientes acciones o caminos que pudieran orientar el sendero de la formación de los investigadores noveles:

\section{Institucionalización de los Semilleros de investigación.}

Una experiencia interesante para la formación de investigadores noveles lo constituye la conformación e institucionalización de los semilleros de investigación. Para ello, se ha considerado la buena práctica que estos han tenido, desde su implantación en 1996, en la 
Universidad de Antioquia (Colombia) hasta la actualidad, a través de la conformación de la Red Colombiana de Semilleros de investigación (REDCOLSI).

Esta es una organización no gubernamental, expresión de un movimiento de científicos de cobertura nacional integrados por estudiantes de educación superior y básica organizados en semilleros de investigación que tratan de fortalecer el proceso de formación y cultura científica de todo el país.

Por lo que antecede, los autores consideran que los semilleros de investigación deben extenderse e institucionalizarse en todas las instituciones educativas, tanto en Latinoamérica como en Colombia, por su relevancia en la formación de investigadores noveles y conformación de comunidades de aprendizaje integrados por estudiantes y profesores reconocidos como investigadores para desarrollar, en conjunto, proyectos que respondan a los objetivos de una línea de investigación institucional o a proyectos sociales de alcance nacional e internacional.

Se comparte, de esta manera, la experiencia de la Universidad de Santo Tomas en Colombia, cuando plantea que los semilleros de investigación deben articularse con las líneas de investigación de la facultad o departamento académico, así como con la estructura curricular de los mismos. De esta manera, los semilleros no deben depender de un docente sino de la facultad o departamento; buscando que los semilleros sean persistentes en el tiempo; sin depender de la permanencia de un docente específico. (Universidad de Santo Tomas, 2017).

En consecuencia, la formación de investigadores noveles debe iniciarse con la conformación de los semilleros como investigación colectiva lo cual le permitirá a estudiantes y a los docentes "aprender a investigar investigando" y de esta manera, manejar, cuestionar y debatir acerca de los conocimientos existentes y formas de concebir la realidad.

\section{Producción de Artículos Científicos.}

Esta acción se deriva del planteamiento anterior, ya que, mediante los semilleros de investigación, los estudiantes trabajan con docentes en función de un proyecto, por tanto, una de las competencias que se debe desarrollar es escribir y reportar los resultados parciales o finales de una actividad de investigación, con lo cual se garantiza la transferencia del conocimiento a la sociedad y se contribuye a la divulgación de los avances producidos en el desarrollo de las investigaciones.

Ello permite que los estudiantes, desde sus inicios, aprendan a fortalecer su proceso de investigación mediante su participación en eventos, congresos, simposios internacionales o nacionales donde deba presentar ponencias o carteles. Estos encuentros con otros investigadores en comunidades científicas garantizan el aprendizaje de la investigación.

Los autores están convencidos que la investigación que no se publica no es investigación, por tanto, se debe incentivar a los estudiantes a reportar sus trabajos, inicialmente en compañía con docentes investigadores o con otros compañeros del semillero.

La producción de artículos científicos reafirma, además, las competencias comunicativas y cognoscitivas que son fundamentales la formación de un investigador a la vez que les permite la búsqueda y el tratamiento de la información mediante el uso de la tecnología (Rodríguez, y Martínez, 2003). Esta competencia es fundamental ya el estudiante no solo recurre a la consulta de diversas fuentes, sino que las organiza, sintetiza y divulga. De allí, la importancia de incentivar a los estudiantes para que publiquen en revistas especializadas arbitradas o indexadas.

Existen en la actualidad revistas especializadas de corte académico en la producción de artículos científicos provenientes de los semilleros; algunas de ellas localizadas en Colombia tales como: Semilleros de Investigación, Cultura investigativa, las cuales pretenden motivar y acompañar la escritura académica de los estudiantes de pregrado y postgrado alrededor de 
proyectos de investigación orientados a proponer alternativas de solución a los problemas del entorno.

\section{Articulación de la Investigación en el Currículo.}

El desarrollo curricular es el eje fundamental de todo el proceso formativo de una institución y este es un proceso en construcción permanente. Por tanto, se plantea articular todas las asignaturas al desarrollo de la investigación de manera que esta no sea un compartimiento estanco que comprende un conjunto de asignaturas o unidades curriculares aisladas, entre ellas la metodología de la investigación, sino por el contrario, la investigación se constituya el eje transversal de todo el proceso formativo ya que desde que inicia el estudiante su carrera o programa inserta su expectativa de proyecto de investigación con todas las asignaturas del plan de estudios, lo que le garantiza las competencias en investigación que son necesarias para abordar la problemática de la realidad con postura teórica y metodológica.

La articulación plantea, además, mirar el currículo bajo la mirada de la inter y transdiciplinariedad, para lo cual se requiere que los docentes trabajen en equipo, de forma colaborativa (Caurcel, García, Rodríguez y Romero, 2009) y tengan flexibilidad y reciprocidad en el uso de las estrategias metodológicas (Dipp, 2013).

Esta concepción curricular conlleva la formación por competencias en investigación y al desarrollo de estructuras del conocimiento mediante un pensamiento complejo, integral y conectado a la realidad (Morin, 1996).

En este sentido, comenta Tobón (2012) que, para facilitar el desarrollo de competencias, es necesario que se articulen, en todas las áreas, actividades de apropiación teórica y de aplicación en torno a problemas, considerando el contexto profesional, científico, social y disciplinar para determinar, a partir de allí, problemas significativos que orienten la formación de los sujetos.

\section{Conclusiones}

La discusión de la temática permitió analizar y argumentar sobre el proceso formativo del investigador novel en educación. Se evidenció que su transitar no es fácil, debido entre otras causas a las dificultades paradigmáticas que tiene la investigación en las ciencias de la educación, producto de las limitaciones propias de su objeto de estudio, el cual es complejo y va más allá de su propia realidad empírica observable para penetrar otro tipo de realidades que son particulares y subjetivas y que influyen en la búsqueda y solución de los problemas socioeducativos.

Por otro lado, la sociedad del conocimiento genera cambios y transformaciones que repercuten en la formación del docente investigador y le permiten, como indica Friere (2011) describir, informar, confrontar y reconstruir procesos de gestión y de solución de problemas en las diferentes ramas, tanto naturales como humanísticas así como asumir procesos de reflexión sobre la acción y desarrollarse como práctico-reflexivo.

Unido a los anterior, la formación del investigador depende también de otros factores como son la escasa valoración del conocimiento, la voluntad política institucional, la cultura académica y la escasa formación que tienen los docentes para enseñar a investigar y la de los estudiantes para tener una actitud positiva hacia el aprendizaje de la investigación.

Se observa, entonces, que la formación de un investigador novel es un proceso gradual que exige dedicación, responsabilidad y compromiso por parte de toda la comunidad académica y de la integración del currículo con la investigación, de manera que esta sea el eje transversal del proceso formativo y mantenga un enfoque inter y transdisciplinario. 
Finalmente, se plantean unas acciones o caminos para la formación integral del individuo y el fortalecimiento de sus competencias en investigación que son: a) institucionalización de los semilleros como espacios para la reflexión, análisis; b) producción de artículos científicos y; c) abordaje de la investigación como eje transversal del currículo.

Las líneas de acción, antes indicadas, podrían ampliarse y complementarse con diferentes experiencias y estudios. Por ello, los autores dejan la puerta abierta para que otros investigadores propongan acciones. Acciones como la conformación de las redes de investigación como instrumentos para generar conocimiento científico de frontera y sus implicaciones en la solución de problemas y transformación de la sociedad así como en la profundización teórica y metodológica de las competencias de investigación en educación que, producto de su complejidad, requiere de diferentes mecanismos para dar respuesta a los problemas de la de la sociedad del conocimiento.

\section{Referencias}

Ander-Egg, E. (2005). Debates y Propuesta sobre la problemática educativa. Algunas reflexiones sobre los retos del futuro. Argentina: Homo Sapiens Editora.

Ander-Egg, E. (1995). Técnicas de Investigación Social. (24ª . Ed.). Argentina: Lumen

Argüelles, A. (1966). Competencia laboral y educación basada en normas de competencia. México: Limusa.

Best, J. (1993). Como Investigar en Educación. (9a ED). España: Morata.

Borja, H., Prada E., Londoño A., Prieto, M., Sanabria, G., Gonzalez, J., Gonzalez, E., \& Gómez, F. (2015). Documento Marco: Investigación. Universidad Santo Tomás. Colombia, Bogotá: Ediciones USTA.

Calderón Hernández, G. (2005). Aprender a investigar investigando. Errores más frecuentes en el proceso investigativo y como evitarlos: Una Aplicación en las ciencias administrativas. Colombia: Universidad Nacional de Colombia, Sede Manizales. Recuperado de: http://www.bdigital.unal.edu.co/3414/1/gregoriocalderonhernandez.2005.pdf

Caurcel, M.J., García, A., Rodríguez, A., \& Romero, A. (2009). ¿Qué opinan los alumnos universitarios sobre las nuevas metodologías activas de enseñanza? Profesorado. Revista sobre currículum y formación del profesorado, 13(1). Recuperado de: https://www.ugr.es/ recfpro/rev131COL2.pdf

Díaz Barriga, F., \& Hernández, G. (2010). Estrategias Docentes para un Aprendizaje Significativo. Una interpretación constructivista. México: Mc Graw Hill.

Dipp, A. (2013). Competencias y Educación. Miradas múltiples de una relación. México: Durango. IUNAES - Radie A.C.

Fallows, S., \& Steven, Ch. (2000). Integrating, key skills in higher education: Employability transferability for a learning life. London: Stylus Publishing.

Freire, P. (2011). La educación como práctica de la libertad. México: Siglo XXI Editores.

Gallego, J.L., \& Rodríguez, A. (2015). Líneas de investigación sobre Educación Especial en España: un estudio bibliométrico (2006-2010). Revista de Ciencias Sociales, 21(2), 219233. Recuperado de: http://digibug.ugr.es/handle/10481/39415

Guido, L. (2012). Aprender a Aprender. México: Tercer Milenio.

Hernández, R., Fernández, C., \& Baptista, L. (2006). Metodología de la Investigación (4ª Ed.). México D. F.: McGraw-Hill Interamericana

Informe final Proyecto Tuning América Latina. (2007) España: Universidad de Deusto. Recuperado de: http://tuningacademy.org/wpcontent/uploads/2014/02/TuningLAIII_Final-Report_SP.pdf

Kerlinger, F. (2002). Investigación del comportamiento. Técnicas y Metodología (4a ed.). México: Mc Graw Hill.

Le Boterf, G. (2001). Ingeniería de las competencias. Barcelona: Gestión 2000 Ediciones

Mardones, J.M. (1991). Filosofía de las ciencias humanas y sociales. Barcelona: Anthropos.

Morín, E. (1998). Introducción al pensamiento complejo. Barcelona: Gedisa Editorial.

Rodríguez, A., \& Martínez, P. (2003). Aplicaciones de la informática a la psicometría en investigación educativa. Comunicar. Revista Científica Iberoamericana de 
Comunicación y Educación, 21, 163-166. Huelva (España). Doi: https://doi.org/10.3916/25566

Rodríguez, A. (2005). Research on special education needs: what and how to investigate in special education. Electronic Journal of Research in Educational Psychology, 5-3(1), 97-112. Recuperado de: psicopedagogica.org/revista/articulos/5/english/Art_5_41a.pdf

Rojas, R. (2008). Formación de investigadores educativos. Una propuesta de investigación. México: Plaza y Valdés S.A. de C.V.

Salvador Mata, F. (2009). Didáctica General. (2 ${ }^{\mathrm{a}}$ Ed.). Madrid: Prentice Hall.

Tobón, S. (2004). Formación Basada en Competencias. Pensamiento Complejo, Diseño curricular y didáctica. Bogotá: Eco.

Tobón, S. (2012). El enfoque socio formativo y las competencias: ejes claves para transformar la educación. En S. Tobón y A. JaikDipp (Coord.), Experiencias de Aplicación de las competencias en la educación y el mundo organizacional (pp. 3-31). Durango, México: Radie.

Universidad Santo Tomás (2017). Lineamientos para registro o actualización de los semilleros de investigación con impacto social. Tunja: Universidad Santo Tomás. Recuperado de: http://investigacion.ustatunja.edu.co/pdf/Lineamientos_semilleros_investigacion.pdf 